\title{
An easy maneuver to screen for moderate-to-severe obstructive sleep spnea: the Simmons Chin Press and Tongue Curl
}

Gerard Joseph Meskill ${ }^{1,2 *}$

Kelly Kincheloe ${ }^{2}$

Jerald Howard Simmons ${ }^{2}$

Sarah Dennis Meskill ${ }^{3}$

${ }^{1}$ Tricoastal Narcolepsy and Sleep Disorders Center, Sleep Medicine Pearland - Texas - United States. ${ }^{2}$ Comprehensive Sleep Medicine Associates, Sleep Medicine - Houston Texas - United States.

${ }^{3}$ Baylor College of Medicine, Pediatric Emergency Medicine - Houston - Texas - United States.
*Corresponding author: Gerard Joseph Meskill

E-mail: gmeskillmd@gmail.com

Received: July 24, 2020;

Accepted: January 8, 2021.

\begin{abstract}
Objectives: To demonstrate that the Simmons chin press and tongue curl (SCPTC) correlates with diagnosis of moderate-to-severe obstructive sleep apnea (msOSA) by itself as well as irrespective of the presence of other associated features. Material and Methods: A consecutive sample of 1,911 sleep studies performed on adult patients from 2/8/2017 to 3/5/2019 was analyzed. The SCPTC exam maneuver was performed on each patient, followed by home sleep apnea testing or in-laboratory polysomnography. The AASM hypopnea 1B 4\% desaturation criteria were utilized for scoring to correlate results to existing literature on morbidity and mortality. A chi-squared using low and high SCPTC score was performed for the outcome of msOSA. Known significant predictors of OSA were dichotomized for comparison and a multiple logistic regression was performed. Results: 1,708 patients qualified for inclusion: 902 males $(52.8 \%)$ and 806 females $(47.2 \%)$ with a mean age of 49.4 and a mean body mass index (BMI) of 28.6. A high SCPTC score correlated with an odds ratio (OR) of $2.49(95 \% \mathrm{CI}: 2.03-3.04, \mathrm{p}<0.001)$ for msOSA. A multiple logistic regression analysis including other risk factors for msOSA demonstrated that high SCPTC scores had an odds ratio for msOSA of 1.77 (95\% CI: 1.40-2.23; p<0.001). Conclusion: The SCPTC is a reproducible physical exam feature that can be utilized by healthcare providers to screen for patients with msOSA.
\end{abstract}

Keywords: Sleep Apnea; Obstructive; Physical Examination; Diagnostic Techniques; Respiratory System; Diagnostic Techniques; Otological; Diagnostic Techniques; Neurological. 


\section{INTRODUCTION}

Untreated obstructive sleep apnea (OSA) can lead to hypertension, coronary artery disease, heart failure, stroke, and insulin resistance and type 2 diabetes $^{1}$. Those experiencing intermittent hypoxemia have an increased all-cause mortality risk $^{1}$. Current prevalence studies estimate that OSA affects $34 \%$ of men and $17 \%$ of women in the US' ${ }^{2}$, while moderate-tosevere OSA (msOSA) affects $13 \%$ of men and $6 \%$ of women ${ }^{3}$.

Since most patients with sleep disorders are evaluated first by their primary care providers (PCPs), the American Academy of Sleep Medicine (AASM) recommends that PCPs screen all high-risk patients, even if asymptomatic ${ }^{4}$. High-risk criteria often are focused on metabolic and cardiovascular (CV) disorders 5 . Other screening tools to identify those at risk rely on symptoms of sleepiness (e.g., Epworth sleepiness scale [ESS]) or characteristic features, such as obesity or snoring volume (e.g., STOP-BANG and Berlin questionnaires).

If OSA were limited to individuals who were overweight, sleepy, or with loud snoring, the aforementioned screening tools might be sufficient. However, these tools underserve patients with atypical features, and studies have demonstrated they are unreliable in predicting OSA in asymptomatic adults ${ }^{6}$. In fact, the majority of OSA disease side effects rest with patients who are asymptomatic since sleepiness (ESS $>10$ ) occurs in only $28 \%$ of moderate and $35 \%$ of severe OSA cases. ${ }^{7}$. Further, significant OSA burden exists in individuals with normal body habitus ${ }^{8,9}$. This becomes critically important when considering the risk of cardiovascular and cerebrovascular morbidity and mortality rests with individuals with OSA under 70 years old, irrespective of symptoms ${ }^{10}$. In females aged 20-70 years old, there is no relationship between OSA and daytime sleepiness ${ }^{11}$. Snoring frequency actually decreases after age 60 , even as OSA prevalence continues to rise ${ }^{12}$. While snoring frequency and severity, excessive daytime sleepiness, male gender, and obesity are the dogma of OSA screening in the community, these observational tools unfortunately leave a significant proportion of the population undiagnosed. US medical training affords little to no formal sleep education ${ }^{13}$, resulting in PCPs being unfamiliar with the effects and long-term ramifications of sleep disorders ${ }^{14}$. These may explain why 80 percent of msOSA cases are thought to be undiagnosed ${ }^{15}$. The financial burden associated with untreated OSA in the US is estimated to be $\$ 148.9$ billion annually secondary to comorbidities and mental health disorders, motor vehicle collisions, workplace accidents, and lost productivity ${ }^{6,16}$.

The Simmons chin press and tongue curl (SCPTC) is a physical exam maneuver performed at the bedside that mimics the position of the mandible and tongue during sleep to assess dynamic effects on upper airway patency during sleep. It is a simple, fast, easily interpreted maneuver that can identify physiologic causes of airway obstruction, regardless of whether they are related to body habitus, excess pharyngeal soft tissue, and/or craniofacial architecture. We aim to demonstrate that it has the ability to identify individuals at risk of msOSA.

\section{MATERIAL AND METHODS}

This is a retrospective cross-sectional study of consecutive adult patients who presented to a sleep practice in an urban setting from $2 / 8 / 2017$ to $3 / 5 / 2019$. Patients were included if they had the SCPTC performed and underwent a diagnostic sleep study. Sleep studies using an oral appliance or for post-surgical assessment were excluded. In agreement with AASM hypopnea 1B criteria, home sleep apnea tests tabulating $3 \%$ rather than $4 \%$ desaturation hypopneas were excluded. The hypopnea $1 \mathrm{~B}$ criteria were selected to correlate the results to existing literature on cardiovascular morbidity risk, which utilized these same criteria. The SCPTC was performed by 1 of 6 practitioners. Each clinician watched a video on how to perform the maneuver.

The SCPTC starts with the patient lying supine with the mouth closed, teeth together, and the temporomandibular joint (TMJ) in an occlusive relation. First, baseline nasal breathing is established. Next, the examiner places gentle pressure on the chin to guide the TMJ into the most retruded centric relation ("chin press"). The patient again is instructed to breathe nasally as the examiner observes for obstruction. The patient then is instructed to touch the tip of the tongue to the hard palate and slide it as posteriorly as possible ("tongue curl") while the examiner repeats the chin press and observes for obstruction. Each maneuver is scored separately: no obstruction scores 0 , partial obstruction 1 , and complete obstruction 2. Partial obstruction is defined as increased resistance to breathing as observed by the patient or the examiner. Complete obstruction is defined as a cessation of airflow despite effort, even if momentarily. The two scores are summed to produce the SCPTC score with 4 total points possible. The SCPTC scores were divided into low (0-2) and high (3-4) categories. Demographic characteristics (age and sex), anthropomorphic data (BMI and neck circumference), and a questionnaire on daytime sleepiness (ESS) were extracted from the medical record. Age was dichotomized to $<50$ years old or $\geq 50$ years old, neck circumference to $<17$ inches or $\geq 17$ inches, BMI to $<35$ or $\geq 35$, and the ESS to $<11$ or $\geq 11$. These cutoffs were chosen to match current screening assessments (i.e., age and BMI correlate to STOP-BANG questionnaire questions; neck circumference of $\geq 17$ inches has been associated with increased risk of $\mathrm{OSA}^{17}$; ESS $\geq 11$ is the established threshold for $\mathrm{EDS}^{18}$ ). An apnea-hypopnea index (AHI) of $\geq 15$ was considered reference standard for msOSA.

A chi-squared using low and high SCPTC score was performed for the outcome of msOSA. In cases with complete data, a multiple logistic regression including known significant predictors of OSA was performed. The multiple logistic model was evaluated for fit by using a receiver operating characteristic (ROC) curve. Data were analyzed using Stata software, version 14.1 (StataCorp). This study was approved by Texas Orthopedic Hospital IRB.

\section{RESULTS}

There were 1,911 consecutive sleep studies. 203 sleep studies were excluded (see Figure 1). The remaining 1,708 patient 
charts were analyzed (Table 1$)$. There were 71 patients (4.1\%) who did not have a neck circumference documented and $13(0.7 \%)$ who did not have an initial ESS documented. In the chi-squared analysis, a high CPTC score had an odds ratio for msOSA of 2.49 (95\% CI: 2.03-3.04, $p<0.001)$. The multiple logistic regression analysis including other risk factors for msOSA demonstrated that a high SCPTC score had an odds ratio of 1.77 (95\% CI: 1.40$2.23, \mathrm{p}<0.001$ ) for msOSA (Table 2).

\section{DISCUSSION}

Undiagnosed OSA is an enormous burden on the US healthcare system, and current screening tools have low sensitivity toward identifying msOSA in patients without metabolic or CV high-risk factors. Individuals with high SCPTC scores have a significant increased risk of msOSA irrespective of the presence of traditional risk factors, such as sleepiness, older age, male sex, obese body habitus, and increased neck circumference. These other features (except sleepiness) all have predictive value in identifying msOSA (see Table 1), but they omit a substantial patient population, and therefore are insufficient for screening.

We suspect the SCPTC's diagnostic power is attributed to the maneuver's reproduction of the retro-position of the tongue and jaw during sleep, thus simulating OSA pathophysiology. The tongue and TMJ muscles exhibit decreased tone during sleep, leading to retro-position of these structures and narrowing of the upper airway. Established exam features (e.g., palate score) rely on static anatomy and do not account for the interrelation between craniofacial anatomy and physiologic changes in musculoskeletal positioning during sleep.

Recently, clinicians have taken interest in analyzing the benefit of treating asymptomatic OSA. One study concluded that treating patients with msOSA did not prevent secondary $\mathrm{CV}$ events, but the subjects in the study had inadequate treatment due to poor compliance (average of 3.3 hours per night), and thus the conclusion is not justified ${ }^{19,20}$. Conversely, Peker et al. $(2016)^{21}$ demonstrated that compliant continuous positive airway pressure (CPAP) use significantly reduced secondary $\mathrm{CV}$ events in non-sleepy OSA patients, while non-compliant use did not. Fu et al. (2017) $)^{22}$ demonstrated that CPAP use reduces allcause mortality in patients with severe OSA.

The SCPTC has the potential to markedly improve detection of OSA. Based upon these results, we recommend any patient with a high SCPTC score to have an evaluation via HSAT or in-laboratory PSG, as the score itself is not sufficient to diagnose msOSA. Sleep disorders screening is limited or absent in most fields of medicine, and therefore dissemination of this technique could have substantial ramifications for secondary disease prevention with subsequent healthcare cost reduction.

\section{CONCLUSION}

This study demonstrates that the SCPTC is a simple physical exam feature that can be utilized by healthcare providers to screen for patients with msOSA.

\section{Limitations}

First, this was a retrospective cross-sectional study with some missing data, though the missing data was minimal and did not affect the overall ability to interpret the regression. Second, this is not a randomized controlled trial, but given this is a screening maneuver that design is not appropriate. Third, the patients analyzed presented due to concerns about a sleep disorder, biasing the study toward those with sleep pathology. Fourth, race and ethnicity were not documented, and therefore we cannot comment on their role on OSA risk.

\section{Author declarations}

The authors have received no financial support for this manuscript. The authors have no conflicts of interest to declare. The study was performed at Comprehensive Sleep Medicine Associates (Houston, Sugar Land, The Woodlands, and Austin, TX).

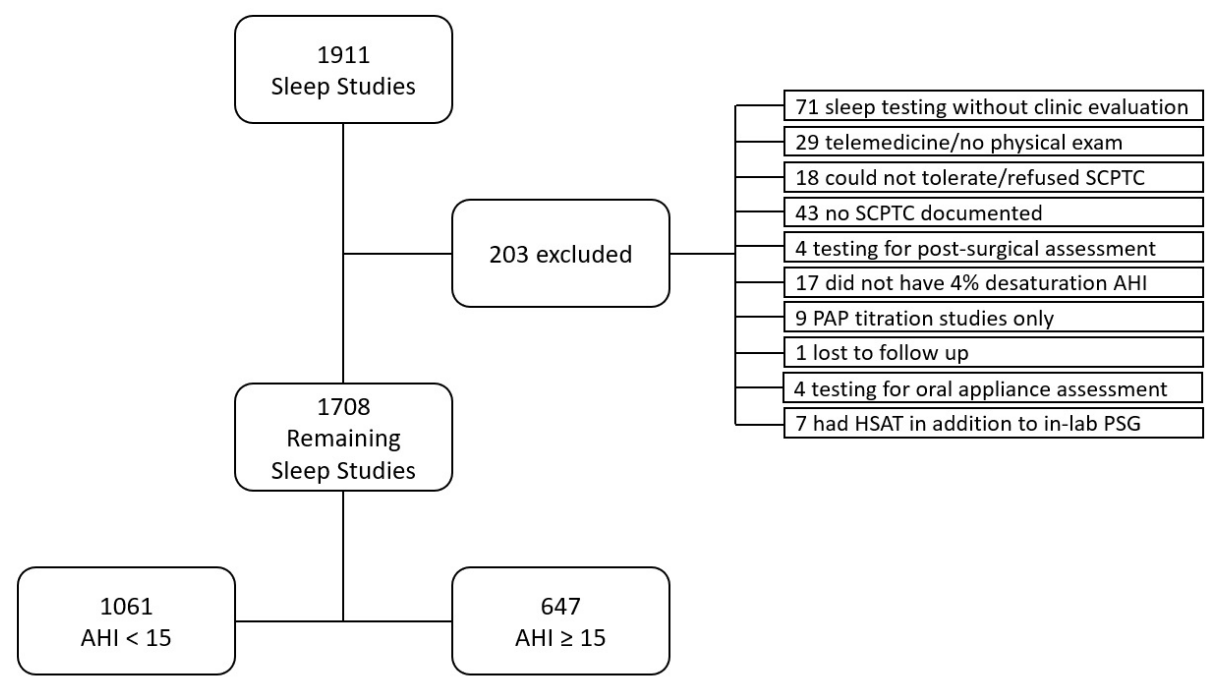

Figure 1. Flow chart of inclusion and exclusion criteria. Notes: SCPTC = Simmons chin press and tongue curl; AHI = Apnea hypopnea index; PAP = Positive airway pressure; HSAT $=$ Home sleep apnea test; PSG $=$ Polysomnogram. 
Table 1. Demographic information on patient population.

\begin{tabular}{lcccc}
\hline & $\begin{array}{c}\text { AHI }<\text { 15 } \\
\text { mean (range) } \\
\mathbf{n = 1 0 6 1}\end{array}$ & $\begin{array}{c}\text { AHI } \geq \mathbf{1 5} \\
\text { mean (range) } \\
\mathbf{n}=\mathbf{6 4 7}\end{array}$ & $\begin{array}{c}\text { Total } \\
\text { (percent)/ } \\
\text { mean }\end{array}$ & p-value \\
\hline Male & 462 & 440 & $902(52.8 \%)$ & $<0.05$ \\
Age (years) & $46.1(18-88)$ & $54.9(21-90)$ & 49.4 & $<0.05$ \\
BMI (kg/m2) & $28.6(15.1-54.1)$ & $34.5(17-82.2)$ & 30.8 & $<0.05$ \\
NC (inches) & $15(10-21.5)$ & $16.9(11.5-24.5)$ & 15.7 & $<0.05$ \\
ESS & $11.3(0-24)$ & $11.1(0-24)$ & 11.2 & 0.49 \\
"High" SCPTC & 228 & 419 & $647(37.9 \%)$ & $<0.05$ \\
score 3-4 & 228 & & &
\end{tabular}

Notes: $\mathrm{BMI}=$ Body mass index $\mathrm{NC}=$ Neck circumference; ESS $=$ Epworth sleepiness scale; SCPTC $=$ Simmons chin press and tongue curl.

Table 2. Logistic regression for moderate-to-severe OSA.

\begin{tabular}{lccc}
\hline & OR & 95\% confidence & $p$-value \\
\hline SCPTC score & 1.77 & $1.40-2.23$ & $<0.001$ \\
ESS $\geq 11$ & 1.11 & $0.88-1.40$ & 0.38 \\
Male sex & 1.87 & $1.43-2.46$ & $<0.001$ \\
Age $\geq 50$ years old & 2.81 & $2.22-3.56$ & $<0.001$ \\
NC $\geq 17$ inches & 2.52 & $1.90-3.33$ & $<0.001$ \\
Body mass index $\geq 35 \mathrm{~kg} / \mathrm{m}^{2}$ & 3.31 & $2.49-4.41$ & $<0.001$ \\
\hline
\end{tabular}

Notes: SCPTC $=$ Simmons chin press and tongue curl; ESS $=$ Epworth sleepiness scale; $\mathrm{NC}=$ Neck circumference.

\section{Informed consent}

This study received IRB exemption. Therefore, no informed consent was required.

\section{ACKNOWLEDGEMENTS}

The authors have no conflicts of interest. We would like to acknowledge Olivia Hua, Kimberly Gonzalez, Maggie Lavender, Krystal May, and Sue Weir for their assistance in collecting data for this manuscript. Additionally, we would like to acknowledge the Fondren Orthopedic Research Institute for their assistance with this study. Finally, we would like to acknowledge Dr. Christian Guilleminault, who was instrumental in the early development of the SCPTC maneuver.

\section{REFERENCES}

1. Punjabi NM, Caffo BS, Goodwin JL, Gottlieb DJ, Newman AB, O'Connor GT, et al. Sleep-disordered breathing and mortality: a prospective cohort study. PLoS Med. 2009 Aug;6(8):e1000132.

2. Gottlieb DJ, Punjabi NM. Diagnosis and management of obstructive sleep apnea: a review. JAMA. 2020 Apr;323(14):1389-400.
3. Peppard PE, Young T, Barnet JH, Palta M, Hagen EW, Hla KM. Increased prevalence of sleep-disordered breathing in adults. Am J Epidemiol. 2013 May;177(9):1006-14.

4. Epstein LJ, Kristo D, Strollo Junior PJ, Friedman N, Malhotra A, Patil SP, et al. Clinical guideline for the evaluation, management and longterm care of obstructive sleep apnea in adults. J Clin Sleep Med. 2009 Jun;5(3):263-76.

5. Aurora RN, Quan SF. Quality measure for screening for adult obstructive sleep apnea by primary care physicians. J Clin Sleep Med. 2016 Aug;12(8):1185-7.

6. Gamaldo C, Buenaver L, Chernyshev O, Derose S, Mehra R, Vana K, et al. Evaluation of clinical tools to screen and assess for obstructive sleep apnea. J Clin Sleep Med. 2018 Jul;14(7):1239-44.

7. Gottlieb DJ, Whitney CW, Bonekat WH, Iber C, James GD, Lebowitz M, et al. Relation of sleepiness to respiratory disturbance index: the sleep heart health study. Am J Respir Crit Care Med. 1999;159(2):502-7.

8. Quintas RD, Novoa MT, Pérez MTA, Martínez MLS, Fernández AA, Velázquez PM. Obstructive sleep apnea in normal weight patients: characteristics and comparison with overweight and obese patients. Arch Bronconeumol. 2013 Dec;49(12):513-7.

9. Gray EL, McKenzie DK, Eckert DJ. Obstructive sleep apnea without obesity is common and difficult to treat: evidence for a distinct pathophysiological phenotype. J Clin Sleep Med. 2017 Jan;13(1):81-8.

10. Franklin KA, Lindberg E. Obstructive sleep apnea is a common disorder in the population - a review on the epidemiology of sleep apnea. J Thorac Dis. 2015 Aug; 7(8):1311-22.

11. Franklin KA, Sahlin C, Stenlund H, Lindberg E. Sleep apnoea is a common occurrence in females. Eur Respir J. 2013 Mar;41(3):610-5.

12. Lindberg E, Taube A, Janson C, Gislason T, Svärdsudd K, Boman G. A 10-year follow-up of snoring in men. Chest. 1998 Oct;114(4):1048-55.

13. Rosen R, Mahowald M, Chesson A, Doghramji K, Goldberg R, Moline M, et al. The taskforce 2000 survey on medical education in sleep and sleep disorders. Sleep. 1998 May;21(3):235-8.

14. Hayes SM, Murray S, Castriotta RJ, Landrigan CP, Malhotra A. (Mis) perceptions and interactions of sleep specialists and generalists: obstacles to referrals to sleep specialists and the multidisciplinary team management of sleep disorders. J Clin Sleep Med. 2012 Dec;8(6):633-42.

15. Lee W, Nagubadi S, Kryger MH, Mokhlesi B. Epidemiology of obstructive sleep apnea: a population-based perspective. Exp Rev Respir Med. 2008 Jun;2(3):349-64.

16. Frost \& Sullivan (US). Hidden health crisis costing America billions: underdiagnosing and undertreating obstructive sleep apnea draining health care system. Mountain View: American Academy of Sleep Medicine; 2016.

17. Davies RJ, Stradling JR. The relationship between neck circumference, radiographic pharyngeal anatomy, and the obstructive sleep apneoa syndrome. Eur Respir J. 1990 May;3(5):509-14.

18. Omobomi O, Quan SF. A requiem for the clinical use of the epworth sleepiness scale. J Clin Sleep Med. 2018 May;14(5):711-2.

19. McEvoy RD, Antic NA, Heeley E, Luo Y, Ou Q, Zhang X, et al. CPAP for prevention of cardiovascular events in obstructive sleep apnea. N Engl J Med. 2016 Sep;375(10):919-31.

20. Chervin RD, Rosen IM, Watson NF. To the editor. N Engl J Med. 2016.

21. Peker Y, Glantz H, Eulenburg C, Wegscheider K, Herlitz J, Thunström E. Effect of positive airway pressure on cardiovascular outcomes in coronary artery disease patients with nonsleepy obstructive sleep apnea. The RICCADSA randomized controlled trial. Am J Respir Crit Care Med. 2016 Sep;194(5):613-20.

22. Fu Y, Xia Y, Yi H, Xu H, Guan J, Yin S. Meta-analysis of all-cause and cardiovascular mortality in obstructive sleep apnea with or without continuous positive airway pressure treatment. Sleep Breath. 2017 Mar;21(1):181-9. 